\title{
Two Results on Discontinuous Input Processing
}

\author{
Vojtěch Vorel ${ }^{1 \star}$ \\ Faculty of Mathematics and Physics, Charles University, Malostranské nám. 25, \\ Prague, Czech Republic, \\ vorel@ktiml.mff.cuni.cz
}

\begin{abstract}
First, we show that universality and other properties of general jumping finite automata are undecidable, which answers a question asked by Meduna and Zemek in 2012. Second, we close the study raised by Černo and Mráz in 2010 by proving that clearing restarting automata using contexts of size two can accept binary non-context-free languages.
\end{abstract}

\section{Introduction}

In 2012, Meduna and Zemek [8,9] introduced general jumping finite automata as a model of discontinuous information processing. A general jumping finite automaton (GJFA) is described by a finite set $Q$ of states, a finite alphabet $\Sigma$, a finite set $R$ of rules from $Q \times \Sigma^{\star} \times Q$, an initial state $q_{0} \in Q$, and a set $F \subseteq Q$ of final states. In a step of computation, the automaton switches from a state $r$ to a state $s$ using a rule $(r, v, s) \in R$, and deletes a factor equal to $v$ from any part of the input word. The choices of the rule used and of the factor deleted are made nondeterministically (in other words, the read head can jump to any position). A word is accepted if there is a computation resulting in the empty word. The boldface term GJFA refers to the class of languages accepted by GJFA. The initial work 89 deals mainly with closure properties of GJFA and its relations to classical language classes (the publications contain flaws, see [13]). It turns out that the class GJFA does not have Boolean closure properties (complementation, intersection) nor closure properties related to continuous processing (concatenation, Kleene star, homomorphism, inverse homomorphism, shuffle). Accordingly, the class also does not stick to classical complexity measures - it is incomparable with both regular and context-free languages. It is a proper subclass of both context-sensitive languages and of the class NP, while there exist NP-complete GJFA languages. See [2], which is an extended version of [3].

On the other hand, the concept of restarting automata [7,10] is motivated by reduction analysis and grammar checking of natural language sentences. In 2010, Černo and Mráz [12] introduced a subclass named clearing restarting automata (cl-RA) in order to describe systems that use only very basic types of reduction

\footnotetext{
* Research supported by the Czech Science Foundation grant GA14-10799S and the GAUK grant No. 52215.
} 
rules (see also 11]). Unlike GJFA, clearing restarting automata may delete factors according to contexts and endmarks, but they are not controlled by state transitions. A key property of each cl-RA is the maximum length of context used. For $k \geq 1$, a $k$-clearing restarting automaton ( $k$-cl-RA) is described by a finite alphabet $\Sigma$ and a finite set $I$ of instructions of the form $\left(u_{\mathrm{L}}, v, u_{\mathrm{R}}\right)$, where $v \in \Sigma^{*}, u_{\mathrm{L}} \in \Sigma^{k} \cup \dot{\zeta} \Sigma^{k-1}$, and $u_{\mathrm{R}} \in \Sigma^{k} \cup \Sigma^{k-1} \$$. The words $u_{\mathrm{L}}, u_{\mathrm{R}}$ specify left and right context for consuming a factor $v$, while $c$ and $\$$ stand for the left and right end of input, respectively.

\section{Preliminaries}

We heavily use the notion of insertion, as it was described, e.g., in [146]:

Definition 1. Let $K, L \subseteq \Sigma^{\star}$ be languages. The insertion of $K$ to $L$ is

$$
L \leftarrow K=\left\{u_{1} v u_{2} \mid u_{1} u_{2} \in L, v \in K\right\} .
$$

More generally, for each $k \geq 1$ we denote

$$
\begin{aligned}
& L \leftarrow^{k} K=\left(L \leftarrow^{k-1} K\right) \leftarrow K, \\
& L \leftarrow^{\star} K=\bigcup_{i \geq 0} L \leftarrow^{i} K,
\end{aligned}
$$

where $L \leftarrow^{0} K$ stands for $L$. In expressions with $\leftarrow$ and $\leftarrow^{\star}$, a singleton set $\{w\}$ may be replaced by $w$.

A chain $L_{1} \leftarrow L_{2} \leftarrow \cdots \leftarrow L_{d}$ of insertions is evaluated from the left, e.g. $L_{1} \leftarrow L_{2} \leftarrow L_{3}$ means $\left(L_{1} \leftarrow L_{2}\right) \leftarrow L_{3}$. Finally, $L \subseteq \Sigma^{\star}$ is a unitary language if $L=w \leftarrow^{\star} K$ for $w \in \Sigma^{\star}$ and finite $K \subseteq \Sigma$.

As described above, a GJFA is a quintuple $M=\left(Q, \Sigma, R, q_{0}, F\right)$. The original definition of the accepted language $L(M)$ is based on configurations that specify a position of the read head (i.e., starting positions of the factor to be erased in the next step). For our proofs, this type of configurations is useless, whence we save space by directly using the following generative characterization [13, Corollary 1] of $L(M)$ as a definition:

Definition 2. Let $M=(Q, \Sigma, R, s, F)$ be a GJFA and $w \in \Sigma^{*}$. Then $w \in \mathrm{L}(M)$ if and only if $w=\epsilon$ and $s \in F$, or

$$
w \in \epsilon \leftarrow v_{d} \leftarrow v_{d-1} \leftarrow \cdots \leftarrow v_{2} \leftarrow v_{1},
$$

where $d \geq 1$ and $v_{1}, v_{2}, \ldots, v_{d}$ is a labeling of an accepting path in $M$.

Definition 3. For $k \geq 0$, a $k$-context rewriting system is a tuple $R=(\Sigma, \Gamma, I)$, where $\Sigma$ is an input alphabet, $\Gamma \supseteq \Sigma$ is a working alphabet not containing the special symbols $\dot{c}$ and $\$$, called sentinels, and $I$ is a finite set of instructions of the form

$$
\left(u_{\mathrm{L}}, v \rightarrow t, u_{\mathrm{R}}\right),
$$


where where $u_{\mathrm{L}}$ is a left context, $x \in \Gamma^{k} \cup \mathrm{\phi} \Gamma^{k-1}, y$ is a right context, $y \in$ $\Gamma^{k} \cup \Gamma^{k-1} \$$, and $v \rightarrow t$ is a rule, $z, t \in \Gamma^{\star}$. A word $w=u_{1} v u_{2}$ can be rewritten into $u_{1} t u_{2}$ (denoted asu $v u_{2} \rightarrow_{R} u_{1} t u_{2}$ ) if and only if there exists an instruction $\left(u_{\mathrm{L}}, v \rightarrow t, u_{\mathrm{R}}\right) \in I$ such that $u_{\mathrm{L}}$ is a suffix of $c u_{1}$ and $u_{\mathrm{R}}$ is a prefix of $u_{2} \$$. The symbol $\rightarrow_{R}^{\star}$ denotes the reflexive-transitive closure of $\rightarrow_{R}$.

Definition 4. For $k \geq 0$, a $k$-clearing restarting automaton ( $k$-cl-RA) is a system $M=(\Sigma, I)$, where $(\Sigma, \Sigma, I)$ is a $k$-context rewriting system such that for each $\mathbf{i}=\left(u_{\mathrm{L}}, v \rightarrow t, u_{\mathrm{R}}\right) \in I$ it holds that $v \in \Sigma^{+}$and $t=\epsilon$. Since $t$ is always the empty word, we use the notation $\mathbf{i}=\left(u_{\mathrm{L}}, v, u_{\mathrm{R}}\right)$. A $k$-cl-RA $M$ accepts the language

$$
L(M)=\left\{w \in \Sigma^{\star} \mid w \vdash_{M}^{\star} \epsilon\right\},
$$

where $\vdash_{M}$ denotes the rewriting relation $\rightarrow_{\bar{M}}$ of $\bar{M}=(\Sigma, \Sigma, I)$. The term $\mathcal{L}(k$-cl-RA) denotes the class of languages accepted by $k$-cl-RA.

Like in GJFA, one may consider the generative approach to languages accepted by clearing restarting automata. In this case, the generative approach is formalized by writing $w_{2} \dashv w_{1}$ instead of $w_{1} \vdash w_{2}$.

\section{Undecidability in General Jumping Finite Automata}

This section proves the following theorem, which solves an open problem stated in 98:

Theorem 5. Given a GJFA $M=(Q, \Sigma, R, s, F)$, it is undecidable whether $L(M)=\Sigma^{*}$.

Proof. Given a context-free grammar $G$ with terminal alphabet $\Sigma_{\mathrm{T}}$, it is undecidable whether $\mathrm{L}(G)=\Sigma_{\mathrm{T}}^{*}[5$. We present a reduction from this problem to the universality of GJFA. Assume that the given grammar $G$ :

- has non-terminal alphabet $\Sigma_{\mathrm{N}}=\left\{A_{1}, \ldots, A_{m}\right\}$ with a start symbol $A_{\mathrm{S}} \in$ $\Sigma_{\mathrm{N}}$,

- does not accept the empty word, and

- is given in Greibach normal form [5] as

$$
B_{i} \rightarrow u_{i},
$$

where $B_{i} \in \Sigma_{\mathrm{N}}$ and $u_{i} \in \Sigma_{\mathrm{T}} \Sigma_{\mathrm{N}}^{*}$ for $i \in\{1, \ldots, n\}$.

We construct a GJFA $M_{G}=(Q, \Gamma, R, s, F)$ as follows, denoting $\Sigma_{\mathrm{B}}=$ $\left\{b_{1}, \ldots, b_{m}\right\}$ :

$$
\begin{aligned}
& Q=\left\{q_{0}, q_{1}, q_{2}, q_{3}, q_{4}\right\}, \\
& \Gamma=\Sigma_{\mathrm{T}} \cup \Sigma_{\mathrm{N}} \cup \Sigma_{\mathrm{B}},
\end{aligned}
$$

$s=q_{0}, F=\left\{q_{4}\right\}$, and $R$ follows Figure 1 Each arrow labeled with a finite set 


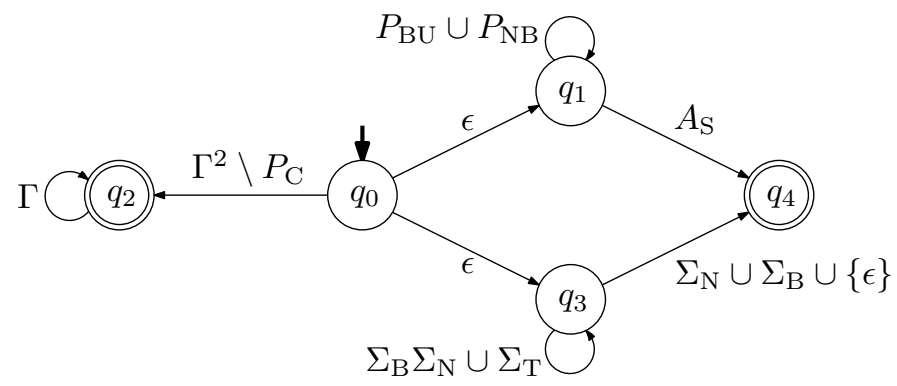

Fig. 1. The GJFA $M_{G}$

$S \subseteq \Gamma^{*}$ stands for $|S|$ transitions, each labeled by a unique $v \in S$. The following finite sets are used:

$$
\begin{aligned}
P_{\mathrm{BU}} & =\left\{b_{i} u_{i} \mid i=1, \ldots, m\right\}, \\
P_{\mathrm{NB}} & =\left\{A_{i} b_{i} \mid i=1, \ldots, m\right\}, \\
P_{\mathrm{C}} & =\left\{x A_{1} \mid x \in \Sigma_{\mathrm{T}}\right\} \cup \\
& \cup\left\{A_{i} b_{i} \mid i=1, \ldots, m\right\} \cup \\
& \cup\left\{b_{i} A_{i+1} \mid i=1, \ldots, m-1\right\} \cup \\
& \cup\left\{b_{m} x \mid x \in \Sigma_{\mathrm{T}}\right\} .
\end{aligned}
$$

For a word $w \in \Gamma^{*}$ we denote with $w_{\mathrm{T}}$ and $w_{\mathrm{N}, \mathrm{B}}$ the projections of $w$ to subalphabets $\Sigma_{\mathrm{T}}$ and $\Sigma_{\mathbf{N}} \cup \Sigma_{\mathbf{B}}$ respectively 1 Let us show that $L(G)=\Sigma_{\mathrm{T}}^{*}$ if and only if $L\left(M_{G}\right)=\Gamma^{*}$.

First, suppose that $L(G)=\Sigma_{\mathrm{T}}^{*}$ and take an arbitrary $w \in \Gamma^{*}$. Describe a derivation of $w_{\mathrm{T}}$ by $G$ using $v_{0}, v_{1}, \ldots, v_{d} \in \Sigma_{\Gamma}, d \geq 1$, where

$$
\begin{aligned}
v_{0} & =A_{\mathrm{S}}, \\
v_{d} & =w_{\mathrm{T}}, \\
v_{k} & =v_{\mathbf{p}, k} A_{i_{k}} v_{\mathbf{s}, k}, \\
v_{k+1} & =v_{\mathbf{p}, k} u_{i_{k}} v_{\mathbf{s}, k}
\end{aligned}
$$

for each $k \in\{0, \ldots, d-1\}$. For $k \in\{0, \ldots, d\}$, we define inductively a word $w_{k} \in \Sigma_{\Gamma}$ satisfying $\left(w_{k}\right)_{\mathrm{T}}=v_{k}$ as follows. First, $w_{0}=A_{\mathrm{S}}$. Next, take $0 \leq k \leq$ $d-1$ and write $w_{k}=w_{\mathbf{p}, k} A_{i_{k}} w_{\mathbf{s}, k}$ such that $\left(w_{\mathbf{p}, k}\right)_{\mathrm{T}}=v_{\mathbf{p}, k}$ and $\left(w_{\mathbf{s}, k}\right)_{\mathrm{T}}=v_{\mathbf{s}, k}$. Then define

$$
w_{k+1}=w_{\mathbf{p}, k} A_{i_{k}} b_{i_{k}} u_{i_{k}} w_{\mathbf{s}, k} .
$$

Informally, the words $w_{0}, \ldots, w_{d}$ describe the derivation of $w_{\mathrm{T}}$ with keeping all the used nonterminals, i.e., $A_{i_{k}}$ is rewrited by $A_{i_{k}} b_{i_{k}} u_{i_{k}}$ instead of $u_{i_{k}}$. Observe

\footnotetext{
${ }^{1}$ A projection to $\Sigma \subseteq \Gamma$ is given by the homomorphism that maps $x \in \Gamma$ to $x$ if $x \in \Sigma$ or to $\epsilon$ otherwise.
} 
that $q_{1} w_{d} \curvearrowright^{*} q_{1} A_{\mathrm{S}}$ using the transitions labeled by words from $P_{\mathrm{BU}}$. Also observe that, due to Greibach normal form, $w_{d} \in\left(\Sigma_{\mathrm{T}} \cup \Sigma_{\mathrm{T}} \Sigma_{\mathrm{N}} \Sigma_{\mathrm{B}}\right)^{*}$, which means that the factors from $\Sigma_{\mathrm{N}} \Sigma_{\mathrm{B}}$ are always separated with letters from $\Sigma_{\mathrm{T}}$.

Distinguish the following cases:

- If $w$ does not have a factor from $\Gamma^{2} \backslash P_{\mathrm{C}}$, all two-letter factors of $w$ belong to $P_{\mathrm{C}}$, which implies that $w$ is a factor of some word from $\left(\Sigma_{\mathrm{T}} t\right)^{*}$, where

$$
t=A_{1} b_{1} A_{2} b_{2} \cdots A_{m} b_{m} .
$$

- If $w$ begins with a letter from $\Sigma_{\mathrm{T}} \cup \Sigma_{\mathrm{N}}$, and ends with a letter from $\Sigma_{\mathrm{T}} \cup \Sigma_{\mathrm{B}}$, then $q_{1} w \curvearrowright^{*} q_{1} w_{d}$ using the transitions labeled by words from $P_{\mathrm{NB}}$. Because $q_{1} w_{d} \curvearrowright^{*} q_{1} A_{\mathrm{S}}$, we conclude $w \in L\left(M_{G}\right)$.

- Otherwise, $w$ starts with a letter from $\Sigma_{\mathrm{B}}$ or ends with a letter from $\Sigma_{\mathrm{N}}$. Then

$$
w_{\mathrm{N}, \mathrm{B}} \in \Sigma_{\mathrm{B}}\left(\Sigma_{\mathrm{N}} \Sigma_{\mathrm{B}}\right)^{*} \cup\left(\Sigma_{\mathrm{N}} \Sigma_{\mathrm{B}}\right)^{*} \Sigma_{\mathrm{N}} \cup \Sigma_{\mathrm{B}}\left(\Sigma_{\mathrm{N}} \Sigma_{\mathrm{B}}\right)^{*} \Sigma_{\mathrm{N}}
$$

and we observe that $q_{0} w \curvearrowright q_{3} w \curvearrowright^{*} q_{3} w_{\mathrm{N}, \mathrm{b}} \curvearrowright u$ for some $u \in \Sigma_{\mathrm{N}} \cup \Sigma_{\mathrm{b}} \cup$ $\{\varepsilon\}$. As $q_{3} u \curvearrowright q_{4}$, we get $w \in L\left(M_{G}\right)$.

- If $w$ has a factor $u \subseteq \Gamma^{2} \backslash P_{\mathrm{C}}$, write $w=w_{\mathbf{p}} u w_{\mathbf{s}}$ and observe

$$
w_{\mathbf{p}} q_{0} u w_{\mathbf{s}} \curvearrowright q_{2} w_{\mathbf{p}} w_{\mathbf{s}} \curvearrowright^{*} q_{2},
$$

implying $w \in L\left(M_{G}\right)$.

Second, suppose that $L\left(M_{G}\right)=\Gamma^{*}$ and take an arbitrary $v=x_{1} x_{2} \cdots x_{n} \in$ $\Sigma_{\mathrm{T}}^{*}$ with $x_{1}, \ldots, x_{n} \in \Sigma_{\mathrm{T}}$. Let $w=\left(x_{1} t\right)\left(x_{2} t\right) \cdots\left(x_{n-1} t\right)\left(x_{n} t\right)$, with $t$ defined in (2). We have $w \in L\left(M_{G}\right)$. Observe that:

- The word $w$ does not contain a factor from $\Gamma^{2} \backslash P_{\mathrm{C}}$.

- By deleting factors from $\Sigma_{\mathrm{B}} \Sigma_{\mathrm{N}} \cup \Sigma_{\mathrm{T}}$, the word $w$ cannot become a word from $\Sigma_{\mathrm{N}} \cup \Sigma_{\mathrm{B}} \cup\{\epsilon\}$.

Thus, $w$ can be accepted by $M$ only using a path through the state $q_{1}$ ending in the state $q_{4}$. In other words, $w$ can be obtained by inserting words from $P_{\mathrm{BU}} \cup P_{\mathrm{NB}}$ to $A_{\mathrm{S}}$. During that process, once an ocurence of some $b_{i}$ fails to be preceded by $A_{i}$, this situation lasts to the very end, which is a contradiction. It follows that $b_{i} u_{i} \in P_{\mathrm{BU}}$ can be inserted only to the right of an occurence of $A_{i}$ that was not followed by $b_{i}$ yet. This corresponds to rewriting $A_{i}$ with $u_{i}$ and we can observe that $w_{\mathrm{T}}=v$ is necessarily generated by the grammar $G$.

\section{Clearing Restarting Automata with Small Contexts}

Though the basic model of clearing restarting automata is not able to describe all context-free languages nor to handle basic language operations (e.g. concatenation and union) [12], it has been deeply studied in order to design suitable generalizations. The study considered also restrictions of the maximum context length to be used in rewriting rules: 


\section{Theorem 6 ([12]).}

1. For each $k \geq 3$, the class $\mathcal{L}(k$-cl-RA) contains a binary language, which is not context-free.

2. The class $\mathcal{L}\left(2\right.$-cl-RA) contains a language $L \subseteq \Sigma^{\star}$ with $|\Sigma|=6$, which is not context-free.

3. The class $\mathcal{L}(k$-cl-RA) contains only context-free languages.

The present section is devoted to proving the following theorem, which completes the results listed above.

Theorem 7. The class $\mathcal{L}(2-\mathrm{cl}-\mathrm{RA})$ contains a binary language, which is not context-free.

In order to prove Theorem [7, we define two particular rewriting systems:

1. A 1-context rewriting system $R_{\mathrm{uV}}=\left(\{\mathrm{u}, \mathrm{V}\},\{\mathrm{u}, \mathrm{V}\}, I_{\mathrm{uV}}\right)$. The set $I_{\mathrm{uV}}$ is listed in Table 1 .

2. A 2-clearing restarting automaton $R_{01}=\left(\{0,1\}, I_{01}\right)$. The set $I_{\mathrm{uV}}$ is listed in Table 2 ,

We write $\rightarrow_{\mathrm{uV}}$ for the rewriting relation of $R_{\mathrm{uV}}$ and $\dashv_{01}$ for the production relation of $R_{01}$.

$$
\begin{array}{|r|r|}
\hline 0) & (\dot{c}, \epsilon \rightarrow \mathrm{uu}, \$) \\
\hline 1) & (\dot{c}, \mathrm{u} \rightarrow \mathrm{uuV}, \epsilon) \\
\hline 2) & (\epsilon, \mathrm{Vu} \rightarrow \mathrm{uuuV}, \epsilon) \\
\hline 3) & (\epsilon, \mathrm{Vu} \rightarrow \mathrm{uuuu}, \$) \\
\hline
\end{array}
$$

\begin{tabular}{|c|c|c|c|c|}
\cline { 2 - 5 } \multicolumn{1}{c|}{} & $(\mathrm{a})$ & $(\mathrm{b})$ & $\mathrm{c})$ & $\mathrm{d})$ \\
\hline 0$)$ & $(\dot{c}, 00, \$)$ & - & - & - \\
\hline 1$)$ & $(\dot{c}, 10,00)$ & $(\mathrm{c}, 00,10)$ & - & - \\
\hline 2$)$ & $(01,10,00)$ & $(00,11,01)$ & $(11,00,10)$ & $(10,01,11)$ \\
\hline 3$)$ & $(01,10,0 \$)$ & $(00,11,0 \$)$ & - & - \\
\hline
\end{tabular}

Table 2. The rules $I_{01}$ sorted by types 0 to 3

The key feature of the system $R_{\mathrm{uV}}$ is:

Lemma 8. Let $w \in L\left(R_{\mathrm{uV}}\right) \cap\{\mathrm{u}\}^{\star}$. Then $|w|=2 \cdot 3^{n}$ for some $n \geq 0$.

The proof is postponed to Section 4.1. We also define:

1. A length-preserving mapping $\varphi:\{0,1\}^{\star} \rightarrow\{\mathrm{u}, \mathrm{V}\}^{\star}$ as $\varphi\left(x_{1} \ldots x_{n}\right)=\bar{x}_{1} \ldots \bar{x}_{n}$, where

$$
\bar{x}_{k}= \begin{cases}\mathrm{V} & \text { if } 1<k<n \text { and } x_{k-1}=x_{k+1} \\ \mathrm{u} & \text { otherwise }\end{cases}
$$

for each $k \in\{1, \ldots, n\}$. 
2. A regular language $K \subseteq\{0,1\}^{\star}$ :

$$
K=\left\{w \in\{0,1\}^{\star} \mid w \text { has none of the factors } 000,010,101,111\right\} .
$$

The following is a trivial property of $\varphi$ and $K$ :

Lemma 9. Let $u \in\{0,1\}^{\star}$. Then $u \in K$ if and only if $\varphi(u) \in\{u\}^{\star}$.

The next lemma expresses how the systems $R_{01}$ and $R_{\mathrm{uV}}$ are related:

Lemma 10. Let $u, v \in\{0,1\}^{\star}$. If $u \dashv_{01} v$, then $\varphi(u) \rightarrow_{\mathrm{uV}} \varphi(v)$.

Proof. For $u=v$ the claim is trivial, so we suppose $u \neq v$. Denote $m=|u|$. As $u$ can be rewrote to $v$ using a single rule of $R_{01}$, we can distinguish which of the four kinds of rules (the rows 0 to 3 of Table 21) is used:

0 ) If the rule 0 is used, we have $u=\epsilon$ and $v=00$. Thus $\varphi(u)=\epsilon$ and $\varphi(v)=u u$.

1) If a rule $\left(\dot{c}, z_{1} z_{2}, y_{1} y_{2}\right)$ of the kind 1 is used, we see that $v$ has some of the prefixes 1000,0010 and so $\varphi(v)$ starts with uuV. Trivially, $\varphi(u)$ starts with u. Because $u[1 .]=.v[3 .$.$] , we have \varphi(u)[2 .]=.\varphi(v)[4 .$.$] and we conclude that$ applying the rule $(\hat{c}, \mathrm{u} \rightarrow \mathrm{uuV}, \epsilon)$ rewrites $\varphi(u)$ to $\varphi(v)$.

$2)$ If a rule $\left(x_{1} x_{2}, z_{1} z_{2}, y_{1} y_{2}\right)$ of the kind 2 is used, we have

$$
\begin{aligned}
& u[k . . k+3]=x_{1} x_{2} y_{1} y_{2}, \\
& v[k . . k+5]=x_{1} x_{2} z_{1} z_{2} y_{1} y_{2} .
\end{aligned}
$$

for some $k \in\{1, \ldots, m-3\}$. As $x_{1} x_{2} y_{1} y_{2}$ equals some of the factors $0100,0001,1110,1011$, we have

$$
\varphi(u)[k+1 . . k+2]=\mathrm{Vu} .
$$

As $x_{1} x_{2} z_{1} z_{2} y_{1} y_{2}$ equals some of the factors $011000,001101,110010,100111$, we have

$$
\varphi(v)[k+1 . . k+4]=\mathrm{uuuV} .
$$

Because $u[. . k+1]=v[. . k+1]$ and $u[k+2 .]=.v[k+4 .$.$] , we have$

$$
\begin{aligned}
\varphi(u)[. . k] & =\varphi(v)[. . k], \\
\varphi(u)[k+3 . .] & =\varphi(v)[k+5 . .] .
\end{aligned}
$$

Now it is clear that the rule $(\epsilon, \mathrm{Vu} \rightarrow \mathrm{uuuV}, \epsilon)$ rewrites $\varphi(u)$ to $\varphi(v)$.

$3)$ If a rule $\left(x_{1} x_{2}, z_{1} z_{2}, y \$\right)$ of the kind 3 is used, we have

$$
\begin{aligned}
u[m-2 . . m] & =x_{1} x_{2} y, \\
v[m-2 . . m+2] & =x_{1} x_{2} z_{1} z_{2} y .
\end{aligned}
$$

As $x_{1} x_{2} y$ equals some of the factors 010,000 , we have

$$
\varphi(u)[m-1 . . m]=\mathrm{Vu} .
$$

As $x_{1} x_{2} z_{1} z_{2} y$ equals some of the factors 01100,00110 , we have

$$
\varphi(v)[m-1 . . m+2]=\mathrm{uuuV} .
$$


Because $u[. . m-1]=v[. . m-1]$, we have

$$
\varphi(u)[. . m-2]=\varphi(v)[. . m-2],
$$

Now it is clear that the rule $(\epsilon, \mathrm{Vu} \rightarrow \mathrm{uuuu}, \$)$ rewrites $\varphi(u)$ to $\varphi(v)$.

Corollary 11. If $u \in L\left(R_{01}\right)$, then $\varphi(u) \in L\left(R_{\mathrm{uV}}\right)$.

Proof. Follows from the fact that $\varphi(\epsilon)=\epsilon$ and a trivial inductive use of Lemma 10.

The last part of the proof of Theorem relies of the following lemma, whose proof is postponed to Section 4.1

Lemma 12. For each $\alpha, \beta>0$ it holds that

$$
00(1100)^{\alpha} 1000(1100)^{\beta} \dashv_{01} 00(1100)^{\alpha+9} 1000(1100)^{\beta-1} .
$$

Corollary 13. For each $\beta>0$ it holds that

$$
001000(1100)^{\beta} \dashv_{01} 00(1100)^{9 \beta} 1000 .
$$

Proof. As the left-hand side is equal to $00(1100)^{0} 1000(1100)^{\beta}$ and the righthand side is equal to $00(1100)^{9 \beta} 1000(1100)^{0}$, the claim follows from an easy inductive use of Lemma 12 ,

Corollary 14. The language $L\left(R_{01}\right) \cap K$ is infinite.

Proof. We show that for each $k \geq 0$,

$$
00(1100)^{\frac{2 \cdot 9^{k}-2}{4}} \in L\left(R_{01}\right) .
$$

In the case $k=0$ we just check that $00 \in L\left(R_{01}\right)$. Next we suppose that the claim holds for a fixed $k \geq 0$ and show that

$$
00(1100)^{\frac{2 \cdot 9^{k}-2}{4}} \dashv_{01} 00(1100)^{\frac{2 \cdot 9^{k+1}-2}{4}} .
$$

Using the rules $1 \mathrm{a}$ and $1 \mathrm{~b}$ we get

$$
00(1100)^{\frac{2 \cdot 9^{k}-2}{4}} \dashv_{01} 1000(1100)^{\frac{2 \cdot 9^{k}-2}{4}} \dashv_{01} 001000(1100)^{\frac{2 \cdot 9^{k}-2}{4}},
$$

while Corollary 13 continues with

$$
001000(1100)^{\frac{2 \cdot 9^{k}-2}{4}} \dashv_{01} 00(1100)^{\frac{2 \cdot 9^{k+1}-18}{4}} 1000 .
$$

Finally, denoting $p=00(1100)^{\frac{2 \cdot 9^{k+1}-18}{4}}$, using rules $2 \mathrm{~b}, 2 \mathrm{a}, 2 \mathrm{~b}, 2 \mathrm{~d}, 2 \mathrm{c}$, and $2 \mathrm{a}$ respectively we get

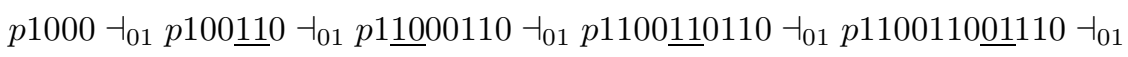

$$
\begin{aligned}
& \dashv_{01} p 1100110011 \underline{0010} \dashv_{01} p 1100110011001 \underline{100}=00(1100)^{\frac{2 \cdot 9^{k+1}-2}{4}} \text {. }
\end{aligned}
$$


We conclude the proof of Theorem 7 by pointing out that Lemmas 9 , 10, and 8 say that for each $w \in\{0,1\}^{\star}$ we have

$$
\begin{aligned}
w \in L\left(R_{01}\right) \cap K & \Rightarrow \varphi(w) \in L\left(R_{\mathrm{uV}}\right) \cap\{\mathrm{u}\}^{\star} \\
& \Rightarrow(\exists n \geq 0)|w|=2 \cdot 3^{n}
\end{aligned}
$$

This, together with the pumping lemma for context-free languages and the infiniteness of $L\left(R_{01}\right) \cap K$, implies that $L\left(R_{01}\right) \cap K$ is not a context-free language. As the class of context-free languages is closed under intersections with regular languages, nor $L\left(R_{01}\right)$ is context-free.

\subsection{Proofs of Lemmas 8 and 12}

Proof (of Lemma 8). We should prove that $w \in L\left(R_{\mathrm{uV}}\right) \cap\{\mathrm{u}\}^{\star}$ implies $|w|=2 \cdot 3^{n}$ for some $n \geq 0$. Let $\Phi:\{\mathrm{u}, \mathrm{V}\}^{\star} \rightarrow \mathbb{N}$ be defined inductively as follows:

$$
\begin{aligned}
\Phi(\epsilon) & =0, \\
\Phi\left(\mathrm{u}^{k} w\right) & =k+\Phi(w), \\
\Phi(\mathrm{V} w) & =1+3 \cdot \Phi(w)
\end{aligned}
$$

for each $k \geq 1$ and $w \in\{\mathrm{u}, \mathrm{V}\}^{\star}$. Observe that we have assigned a unique value of $\Phi$ to each word from $\{\mathrm{u}, \mathrm{V}\}^{\star}$. Next, we describe effects of the rules of $R_{\mathrm{uV}}$ to the value of $\Phi$.

$0)$ The rule 0 can only rewrite $w_{1}=\epsilon$ to $w_{2}=u u$. We have $\Phi\left(w_{1}\right)=0$ and $\Phi\left(w_{2}\right)=2$.

1) The rule 1 rewrites $w_{1}=\mathrm{u} w$ to $w_{2}=\mathrm{uuV} w$ for some $w \in\{\mathrm{u}, \mathrm{V}\}^{\star}$. We have $\Phi\left(w_{1}\right)=1+\Phi(w)$ and $\Phi\left(w_{2}\right)=3+3 \cdot \Phi(w)$. Thus, $\Phi\left(w_{2}\right)=3 \cdot \Phi\left(w_{1}\right)$.

2) The rule 2 rewrites $w_{1}=\bar{w} \mathrm{Vu} w$ to $w_{2}=\bar{w} \mathrm{uuuV} w$ for some $w, \bar{w} \in\{\mathrm{u}, \mathrm{V}\}^{\star}$. We have

$$
\Phi(\mathrm{Vu} w)=\Phi(\mathrm{uuuV} w)=4+3 \cdot \Phi(w) .
$$

It follows that $\Phi\left(w_{1}\right)=\Phi\left(w_{2}\right)$.

3) The rule 3 rewrites $w_{1}=\bar{w} \mathrm{Vu}$ to $w_{2}=\bar{w}$ uuuu for some $\bar{w} \in\{\mathrm{u}, \mathrm{V}\}^{\star}$. We have $\Phi(\mathrm{Vu})=\Phi(\mathrm{uuuu})=4$ and thus $\Phi\left(w_{1}\right)=\Phi\left(w_{2}\right)$.

Together, each $w \in L\left(R_{\mathrm{uV}}\right)$ has $\Phi(w)=2 \cdot 3^{n}$ for some $n \geq 0$. As $\Phi(w)=|w|$ for each $w \in\{\mathrm{u}\}^{\star}$, the proof is complete.

Proof (of Lemma 12). We should prove that

$$
00(1100)^{\alpha} 1000(1100)^{\beta} \dashv_{01} 00(1100)^{\alpha+9} 1000(1100)^{\beta-1} .
$$


for each $\alpha, \beta>0$. Indeed:

$$
\begin{aligned}
& 00(1100)^{\alpha} 1000(1100)^{\beta} \dashv_{01} 00(1100)^{\alpha} 100 \underline{110}(1100)^{\beta} \quad \dashv_{01}
\end{aligned}
$$

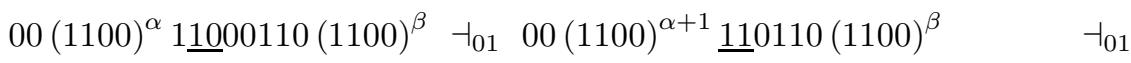

$$
\begin{aligned}
& 00(1100)^{\alpha+1} 110 \underline{01110}(1100)^{\beta} \dashv_{01} 00(1100)^{\alpha+2} 1110 \underline{01}(1100)^{\beta} \quad \dashv_{01} \\
& 00(1100)^{\alpha+2} 11 \underline{001001}(1100)^{\beta} \dashv_{01} 00(1100)^{\alpha+3} 1 \underline{10001}(1100)^{\beta} \quad \dashv_{01} \\
& 00(1100)^{\alpha+4} \underline{1101}(1100)^{\beta} \dashv_{01} 00(1100)^{\alpha+4} 11011 \underline{00100}(1100)^{\beta-1} \dashv_{01} \\
& 00(1100)^{\alpha+4} 110 \underline{011100100}(1100)^{\beta-1} \dashv_{01} 00(1100)^{\alpha+5} 11 \underline{00100100}(1100)^{\beta-1} \dashv_{01} \\
& 00(1100)^{\alpha+6} 1 \underline{1000100}(1100)^{\beta-1} \dashv_{01} 00(1100)^{\alpha+7} 01 \underline{1000}(1100)^{\beta-1} \quad \dashv_{01}
\end{aligned}
$$

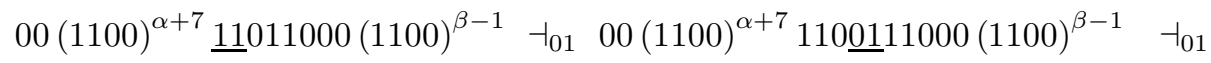

$$
\begin{aligned}
& 00(1100)^{\alpha+8} 11 \underline{001000}(1100)^{\beta-1} \text {. }
\end{aligned}
$$

\section{References}

1. Ehrenfeucht, A., Haussler, D., Rozenberg, G.: On regularity of context-free languages. Theoretical Computer Science 27(3), 311 - 332 (1983)

2. Fernau, H., Paramasivan, M., Schmid, M.L.: Characterization and complexity results on jumping finite automata. submitted to Theoretical Computer Science (2015)

3. Fernau, H., Paramasivan, M., Schmid, M.L.: Jumping finite automata: Characterizations and complexity. In: Drewes, F. (ed.) Implementation and Application of Automata, Lecture Notes in Computer Science, vol. 9223, pp. 89-101. Springer International Publishing (2015)

4. Haussler, D.: Insertion languages. Information Sciences 31(1), 77 - 89 (1983)

5. Hopcroft, J.E., Motwani, R., Ullman, J.D.: Introduction to automata theory, languages, and computation, 2nd edition. Addison-Wesley (2003)

6. Ito, M., Kari, L., Thierrin, G.: Insertion and deletion closure of languages. Theoretical Computer Science 183(1), 3 - 19 (1997)

7. Jančar, P., Mráz, F., Plátek, M., Vogel, J.: Restarting automata. In: Reichel, H. (ed.) Fundamentals of Computation Theory, Lecture Notes in Computer Science, vol. 965, pp. 283-292. Springer Berlin Heidelberg (1995)

8. Meduna, A., Zemek, P.: Jumping finite automata. International Journal of Foundations of Computer Science 23(7), 1555-1578 (2012)

9. Meduna, A., Zemek, P.: Regulated Grammars and Automata. Springer US (2014), chapter 17: Jumping Finite Automata

10. Mráz, F., Plátek, M., Vogel, J.: Restarting automata with rewriting. In: Jeffery, K., Král, J., Bartošek, M. (eds.) SOFSEM'96: Theory and Practice of Informatics, Lecture Notes in Computer Science, vol. 1175, pp. 401-408. Springer Berlin Heidelberg (1996)

11. Černo, P.: Clearing restarting automata and grammatical inference. In: Jeffrey Heinz, Colin de la Higuera, T.O. (ed.) Proceedings of the Eleventh International Conference on Grammatical Inference. JMLR Workshop and Conference Proceedings, vol. 21, pp. 54-68 (2012)

12. Černo, P., Mráz, F.: Clearing restarting automata. Fundamenta Informaticae 104(1), 17-54 (2010) 
13. Vorel, V.: On basic properties of jumping finite automata. International Journal of Foundations of Computer Science (conditionally accepted) (2015) 